\title{
The Qualitative Hyperalgesia Profile: A New Metric to Assess Chronic Post-Thoracotomy Pain
}

\author{
Jeffrey Chi-Fei Wang ${ }^{1}$, Ching-Hsia Hung ${ }^{1,2}$, Peter Gerner ${ }^{3}$, Ru-Rong Ji ${ }^{4}$ and Gary R. Strichartz ${ }^{1, *}$ \\ ${ }^{I}$ Pain Research Center, Brigham \& Women's Hospital, Boston MA 02115, USA; ${ }^{2}$ Department of Physical Therapy, \\ Medical College, National Cheng Kung University, Tainan, R.O.C. Taiwan; ${ }^{3}$ Department of Anesthesia, University of \\ Salzburg, Salzburg, Austria; ${ }^{4}$ Department of Anesthesiology, Duke University, Durham, NC, UK
}

\begin{abstract}
Thoracotomy often results in chronic pain, characterized by resting pain and elevated mechano-sensitivity. This paper defines complex behavioral responses to tactile stimulation in rats after thoracotomy, shown to be reversibly relieved by systemic morphine, in order to develop a novel qualitative "pain" score. A deep incision and 1 hour of rib retraction in male Sprague-Dawley rats resulted in reduced threshold and a change in the locus of greatest tactile (von Frey filament) sensitivity, from the lower back to a more rostral location around the wound site, and extending bilaterally. The fraction of rats showing nocifensive responses to mild stimulation $(10 \mathrm{gm})$ increased after thoracotomy (from a preoperative value of $0 / 10$ to $8 / 10$ at 10 days post-op), and the average threshold decreased correspondingly, from $15 \mathrm{gm}$ to $\sim 4 \mathrm{gm}$. The nature of the nocifensive responses to tactile stimulation, composed pre-operatively only of no response (Grade 0 ) or brief contractions of the local subcutaneous muscles (Grade I), changed markedly after thoracotomy, with the appearance of new behaviors including a brisk lateral "escape" movement and/or a $180^{\circ}$ rotation of the trunk (both included as Grade II), and whole body shuddering, and scratching and squealing (Grade III). Systemic morphine (2.5 $\mathrm{mg} / \mathrm{kg}$, i.p.) transiently raised the threshold for response and reduced the frequency of Grade II and III responses, supporting the interpretation that these represent pain. The findings support the development of a Qualitative Hyperalgesic Profile to assess the complex behavior that indicates a central integration of hyperalgesia.
\end{abstract}

Keywords: Post-operative, allodynia, morphine, mechano-sensitivity, preventive analgesia.

\section{INTRODUCTION}

Despite much research, our knowledge of the mechanisms of chronic post-operative pain remains limited [1-6]. Peripheral factors that are released by damaged tissue have identified importance for the induction of acute postincisional pain [7-10], and a role for central glutamate receptors [11, 12], TRPV1 [13], and certain mitogen activated protein kinases (MAPK), such as p38 [14, 15], in both glia and neurons [15-18], have been shown for the induction of acute post-incisional and of prolonged, retraction-induced pain [19]. However, there have been almost no studies on the factors that are critical for the pain that persists for months after surgery.

Estimates of patients suffering pain lasting for more than several months following thoracotomy range as high as $40 \%$ $[1,20-25]$. Pain occurs at rest, i.e. with shallow breathing, and also has a pronounced movement-related component during coughing, stretching or twisting, an example of mechano-hyperalgesia [26-28]. Debate has centered around the frequency with which this pain can be classified as neuropathic [22, 29-31] and intentional modifications in surgical procedures have reduced the incidence of chronic pain $[28,32-36]$, primarily by lowering the likelihood of intercostal nerve damage [37-41]. A variety of local and

*Address correspondence to this author at the Pain Research Center, Brigham \& Women's Hospital, Boston MA 02115, USA;

Tel: 617 732-7802/-8797; Fax: 617 730-2801;

Emails: gstrichartz@partners.org and gstrichz@zeus.bwh.harvard.edu other anesthetic methods have also been shown to reduce post-thoracotomy pain [24, 42-46], but the mechanisms of effect here are not obvious since such drugs are known to act on multiple different targets [47]. At present the cellular and molecular mechanisms, for the most part assigned to the central nervous system $[1,17,31,48]$, that result in the persistence of such pain are obscure.

An important opportunity to approach this problem was provided by the development of a model for chronic postthoracotomy pain in the rat [49]. This first report described the surgical procedure, reported mechanical hypersensitivity and cold allodynia, characterized their time course, and showed evidence of peripheral nerve injury at the surgical site. An earlier paper showed that intercostal nerve ligation caused tactile hypersensitivity lasting at least one month, whereas cutting the nerve cleanly caused only two weeks of hypersensitivity [50]. A number of "escape" behaviors, such as jumping, scratching the site, squeaking and trembling were observed from tactile stimulation with von Frey hairs in the nerve ligated rats, but only a summed score from these behaviors was reported. No such analysis of rats after thoracotomy has been reported.

Others have used the thoracotomy model to examine the preventive actions of systemic local anesthetic [51], and the ineffectiveness of nerve treatment with resiniferatoxin [52]. As with much other work on experimental animal "pain," these and the above-mentioned reports used rapid withdrawal responses to brief external stimulation to 
quantify "nocifensive" behavior. In the absence of measurements of central processing, such as fMRI or other brain imaging methods, we cannot be certain that such withdrawal corresponds to CNS responses to perceived pain. Indeed, the possibility that withdrawal does not equate with pain (but perhaps to some other sensation/perception) raises the concern that agents that are effective as "antihypersensitivity" treatments in animal models may very likely fail as effective clinical analgesics [2, 53, 54].

In the present work we have used the rat thoracotomy model to identify complex evoked behaviors for measuring post-operative "pain." Beyond measuring the frequency and time-course of simple withdrawal responses to tactile stimuli, we have identified and graded more complex behavior, including turning of the body, a whole-body tremble or scratching and squealing, as a means of registering a degree of perceived pain. Post-thoracotomy rats have also been administered systemic morphine for its known analgesic actions, in order to validate the identification of these complex behaviors with actual pain [55].

In addition, the dorsal root ganglia (DRG) have been removed from hypersensitive rats after thoracotomy for double immunostaining to detect the expression of ATF3, a known marker of nerve injury [56], and NF-200, a known marker for myelinated neurons. Our results reveal the presence of nerve injury (ATF-3 expression) by thoracotomy in both myelinated and non-myelinated neuronal types.

\section{MATERIALS AND METHODS}

\section{Animals}

All procedures were approved by the Harvard Medical Area Standing Committee on Animals (Boston, MA) and are in keeping with international standards for the care and treatment of laboratory animals [57] Male Sprague-Dawley rats were purchased from Charles River Laboratory (Wilmington, MA) and kept in the animal housing facilities at Brigham and Women's Hospital, with controlled relative humidity $(20 \%-30 \%)$, at room temperature $\left(24^{\circ} \mathrm{C}\right)$, and under a $12-12 \mathrm{~h}$ light-dark cycle, with free access to food and water. They were handled for 5-7 days before the procedure to familiarize them with the experimental environment, so as to minimize stress-induced analgesia and to establish baseline behavioral parameters for each individual animal. At the time of surgery, animals weighed 280-310 g.

\section{Surgical Procedures}

Endotracheal Intubation. Rats were briefly anesthetized with 4\%-5\% sevoflurane (Sevorane, Abbott Laboratory, North Chicago, IL, USA) before receiving intraperitoneal pentobarbital sodium $(60 \mathrm{mg} / \mathrm{kg}$; Nembutal, Akorn, Inc., Lake Forest, IL). Animals were then tracheally intubated by a method modified from Weksler et al. [58]. The anesthetized rat was placed in the supine position with a small pillow under the neck. An otoscope (Welch Allyn, Inc., Skaneateles Falls, NY) with a number 3 speculum was introduced into the animal's oropharynx, and the tongue was gently retracted and fixed above the speculum by left index finger compression. A guide wire (spring-wire guide: 0.46 mm dia. X 25 cm; Arrow ${ }^{\circledR}$ International, Inc., Reading, PA) was introduced through the epiglottis, then vocal cords, into the trachea. The otoscope removed over the wire, a 16-gauge polyethylene Angiocath $\left(1.7 \quad \mathrm{X} \quad 51 \quad \mathrm{~mm}\right.$ Insyte $^{\mathrm{TM}}$ Autoguard $^{\mathrm{TM}}$ winged; BD Infusion Therapy Systems Inc., Sandy, UT) was glided over the wire to its full length. The wire was removed, the catheter was connected to a Yconnector attached to tubing from a small animal pressure controlled ventilator (model TOPO220; Kent Scientific Corporation, Torrington, $\mathrm{CN}$ ), which was set at a respiratory rate of $65-80 / \mathrm{min}$. An isoflurane vaporizer (SurgiVet) was connected to the intake of the ventilator to deliver a concentration $1.0 \%-1.5 \%$ isoflurane in oxygen when necessary. $\mathrm{A} \mathrm{CO}_{2}$ analyzer (model CapStar-100; IITC Inc., Woodland Hills, CA) was connected to the expiratory end to monitor end tidal $\mathrm{CO}_{2}$, which was maintained at $25-40 \mathrm{~mm}$ $\mathrm{Hg}$ for the entire surgical procedure.

Thoracotomy and Rib Retraction. Following closely the report of Buvanendran et al. [49], the anesthetized rats were placed in the left decubitous position with a pillow under the contralateral armpit to elevate the surgical field. The skin below the ear line and above the superior iliac crest was shaved on both sides. A $3 \mathrm{~cm}$ long incision was made in the skin of the right lateral chest wall along the fourth intercostal line, beginning from $1 \mathrm{~cm}$ lateral to the midline and $1 \mathrm{~cm}$ below the inferior angle of right scapula (Fig. 1). The superficial and deep lateral thoracic muscles covering the ribs were incised and retracted to expose the intercostal muscles. A $1.0 \mathrm{~cm}$ incision was made through the intercostal muscle and pleura along the cranial border of the fifth rib. The blunt tines of a small retractor (model 17003-03, Goldstein $3 \times 3$ sharp teeth with depth $4.5 \mathrm{~mm}$, teeth width 6.5 $\mathrm{mm}$; FST, Inc., Foster City, CA) were placed under the fourth and fifth ribs. The retractor was opened to separate the ribs by $1 \mathrm{~cm}$, and was left in place for $60 \mathrm{~min}$, as previously described [49]. During this time the open wound was covered with wet-dressing gauze kept moist with sterile phosphate buffered saline (PBS). After one hour the retractor was closed and removed and the fourth and fifth ribs were approximated and ligated tightly with 4-0 Vicryl sutures (Ethicon, Sommerville NJ). Air was aspirated from the pleural cavity with a 5-mL syringe attached to the polyethylene tubing to restore normal intrapleural pressure. The superficial muscle covering the ribs was then apposed with 4-0 Vicryl sutures (MYCO Medical, Cary, NC), and the skin was closed with 3-0 prolene sutures (Ethicon). The animals were allowed to recover, and the endotracheal catheter was removed once spontaneous breathing was reestablished.

\section{Drugs}

Morphine sulfate, USP grade, was purchased from WestWard Pharmaceuticals (Eatontown, NJ) and a stock solution of $2.5 \mathrm{mg} / \mathrm{mL}$ made by dissolving in sterile saline. Different volumes of this stock solution were injected intraperitoneally with a $28 \mathrm{~g}$ needle attached to a $1 \mathrm{cc}$ insulin syringe to achieve a final dose of $2.5 \mathrm{mg} / \mathrm{kg}$. No signs of ataxia, locomotion or imbalance were detected after this dosing, and normal exploratory behavior was not altered, suggesting that no major motor deficits were present. Consistent with this, comparisons of brain activity during morphine analgesia with that from known sedatives that have little to no analgesic activity have shown that even at $3 \mathrm{mg} / \mathrm{kg}$ i.p., morphine has little sedative effect [55]. 


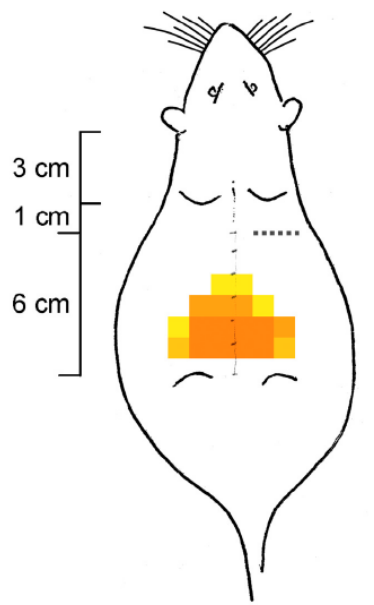

Baseline

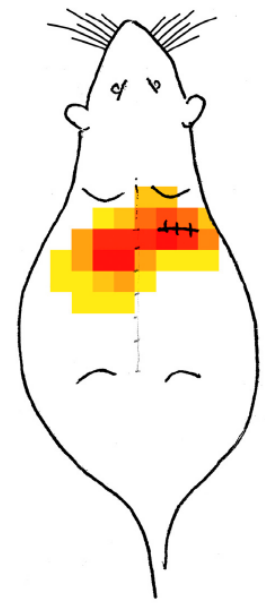

POD 5

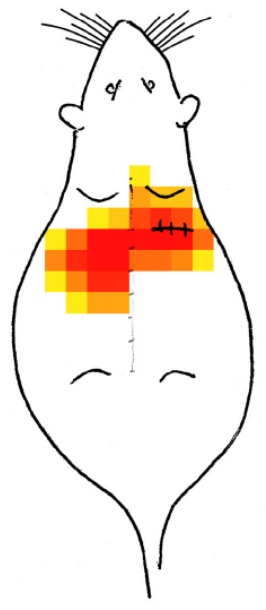

POD 14

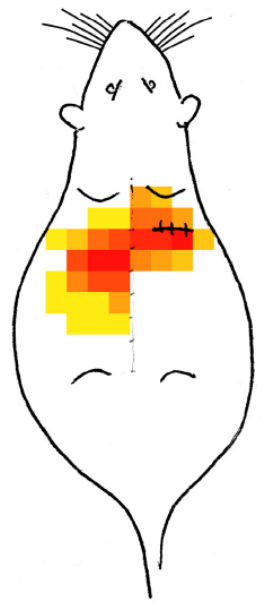

POD 36

Fig. (1). Map of tactile sensitivity on the rat's back before and at 5, 14 and 36 days after thoracotomy and rib retraction. The dashed line caudal to the right shoulder blade shows the location of the incision-retraction. The color of each pixel, a $1 \mathrm{~cm} X 1 \mathrm{~cm}$ area, indicates the number of rats, in the total of 8 that were operated on, that responded to the lightest von Frey filament (VFH) at the indicated location. Grey tone: single responding rat; yellow to red: increasing numbers of responders, from 2 to 8 , respectively. Note how the most sensitive area shifts from a more caudal and nearly bilaterally symmetric location in the pre-operative, baseline animals to a location around the surgical site, and extending more laterally than before the operation.

\section{Behavioral Testing}

To test mechanical hyperalgesia, each rat was placed in a loose restraining cage $(8 \mathrm{~cm} \mathrm{X} 9 \mathrm{~cm} \times 20 \mathrm{~cm})$ and allowed to rest there for 15 minutes. A series of calibrated von Frey filaments (VFH; Stoelting Co, Wood Dale, IL) with bending forces ranging from 0.4 to $15.0 \mathrm{~g}$ was applied perpendicularly to the dorsal skin surface, starting from the lowest force, to calculate the threshold for a nocifensive response. Each VFH was applied sequentially at a particular rostro-caudal region, starting at the contralateral side, moving towards the midline, and then extending to the ipsilateral area (Fig. 1). By this process the skin was tested along bands of $1 \mathrm{~cm}$ width at each dorso-thoracic segment, moving from the least to the most sensitive area. Each testing spot, separated by $1 \mathrm{~cm}$, was probed twice, pressing with a 3 second duration spaced 3 seconds apart. There was a 30 second resting period between each spot tested. The VFH force was increased progressively until a defined response occurred (see below) and then reduced and again increased to verify the threshold force. Rats responding to the lowest force filament that was used, $0.4 \mathrm{~g}$, were assigned this as the threshold, and those not responding to the highest force were assigned a "ceiling" threshold of $15.1 \mathrm{~g}$. Higher forces were avoided to minimize the tactile sensitization that occurs with these stiffer VFHs.

In one analysis of threshold for nocifensive responses, the average threshold force, to produce any response, was calculated for each experimental group. In a different analysis, "allodynia" was functionally defined by any response to a $10 \mathrm{~g} \mathrm{VFH}$ since that force never caused a response in handled animals in the preoperative period; the fraction of rats in each group that responded to a $10 \mathrm{~g} \mathrm{VFH}$ was then used as an allodynic population fraction (0-1.0).

Nocifensive behavior was categorized by the intensity of response into four grades, according to the behavior displayed, as follows: Grade 0 - no response, even at $15 \mathrm{~g}$ VFH stimulation; Grade I - slight movement of feet to shift body away from the stimulus or local subcutaneous muscle contraction (the cutaneous truncii muscle response, CTMR) [59]; Grade II - strong CTMR and brisk lateral movement of the trunk by several centimeters away from the stimulus, followed by turning around $\left(180^{\circ}\right.$ re-orientation); Grade III - behavior of Grade II plus scratching near the test location, whole body shuddering or vocalization. In naïve, unoperated rats Grade II and III behavior were rarely if ever observed when poking was done with VFH up to $26 \mathrm{gm}$, although these behaviors, except for the shudder, were observed in response to a forceful pinch of the lumbar back skin with serrated forceps. Thus, in a third type of analysis each experimental group was divided according to the fraction of animals that showed Grade I, Grade II or Grade III at threshold. The distribution of the population among these four grades is termed the Qualitative Hyperalgesia Profile.

The responsive area over which any of these ostensibly nocifensive behaviors was first induced by the weakest VFH, i.e., at the threshold for the least intense nocifensive response, was mapped for each individual rat. Responsive areas for each rat in the test cohort (usually $n=7-8$ ) were graphically summed over all rats to give a "color map" showing the anatomical distribution of post-operative hypersensitivity all rats in the experimental group (Fig. 1).

Behavioral evaluations were made over 2 days before thoracotomy, and averaged as the baseline, and then daily at post-operative days (PODs) $1-8$, then every 2 days until day 36 , at which time animals were euthanized by $\mathrm{CO}_{2}$ asphyxiation.

Dorsal Root Ganglion Immunocytochemistry. All animals were terminally anesthetized with isoflurane $(>5 \%)$ and perfused through the ascending aorta with saline followed by $4 \%$ paraformaldehyde containing $1.5 \%$ picric acid and $0.16 \mathrm{M} \mathrm{PB}\left(\mathrm{pH} 7.4,4^{0} \mathrm{C}\right.$, Ji et al., 1995). After perfusion, DRGs were removed and post-fixed in the same fixative overnight. DRG sections $(15 \mu \mathrm{m})$ were cut on a 
cryostat. For single immunofluorescence, sections were blocked with $2 \%$ goat serum in $0.3 \%$ Triton for $1 \mathrm{~h}$ at room temperature (RT), and incubated for $16-48 \mathrm{~h}$ at $4{ }^{\circ} \mathrm{C}$ with primary antibody. DRG sections were stained with the following primary antibodies: anti-ATF-3 (1:1000, rabbit, Santa Cruz) and NF-200 (1:2000, mouse, Chemicon). Sections will then be incubated with FITC- or CY3conjugated secondary antibody (1:400, Jackson Immunolab) for 60-90 minutes. For double immunofluorescence, sections were incubated with a mixture of primary antibodies from different species (e.g., rabbit and mouse primary antibodies), followed by a mixture of FITC-conjugated and CY3conjugated secondary antibodies [60, 61]. Immunostained slides were examined under a Nikon fluorescence microscope. All the images were captured with a high resolution CCD Spot camera (Diagnostic Instruments Inc.) under the same conditions and analyzed with NIH Image software or Adobe PhotoShop. To determine co-localization after double staining, two single stained images were merged under Adobe PhotoShop, and single and double-labeled cells were detected from the merged images.

\section{Statistical Analysis}

Threshold forces are presented as mean \pm SEM, and are compared for statistically significant differences among groups by multi-group ANOVA followed by Tukey's test. When compared to the pre-operative baseline value or to the post-operative + pre-morphine value, these analyses are corrected for repeated measures adjustments. The behavior in the QHP analysis is reported as the population distribution among the different grades in any one treatment group; significant differences among distributions in the different groups were determined by Jonckheere-Terpstra statistics. All statistical tests were conducted using SAS version 9.3 software (SAS, Cary, N.C.). Unless specifically noted, significance occurred for $\mathrm{P}<0.05$.

\section{RESULTS}

Anatomical distribution of tactile hypersensitivity changes after thoracotomy. Before thoracotomy, the area of lowest threshold was centered around the midline, near the L3-L5 vertebra and extending $2-4 \mathrm{~cm}$ bilaterally (Fig. 1, Baseline). Most of the rats showed no response, even to a $15 \mathrm{~g} \mathrm{VFH}$, the maximum force used, and none had their lowest thresholds at the site of future incision/retraction, shown by the dotted line in the "Baseline" schematic of (Fig. 1).

The initial increase in tactile hypersensitivity was not observed until 3-4 days after surgery (post-operative day 3-4, POD3-4), from which time it became progressively worse (Fig. 2). On POD8 almost all the rats showed some nocifensive response to VFHs below $10 \mathrm{~g}$ (Fig. 2A), and the most sensitive area was located more rostrally, on the surgical (ipsilateral) side, close to the incision/retraction site (Fig. 1). It is noteworthy that hypersensitivity was also present on the contralateral side, centered about $1 \mathrm{~cm}$ more caudal than the most sensitive locus to ipsilateral stimulation (Fig. 1, POD14). There was a concomitant lateral spread in the responsive area, both ipsi- and contralateral. At this time most of the rats responded to at least one of the filaments of $15 \mathrm{~g}$ or less, as shown by the red-orange zones of the pain "color map" (Fig. 1). By POD14 the area of pain responsiveness had increased slightly and the hypersensitive population fraction had reached the maximum (7-8/1C; Fig. $\mathbf{2 A})$, although the overall anatomical distribution was centered on the same region as on POD5. At POD36 the area of pain had regressed slightly, but the same fraction of rats remained hypersensitive as at POD14 (Fig. 2A).

Thoracotomy reduces threshold for nocifensive responses. Thoracotomy and rib retraction was also followed after POD3-4 by a drop in threshold for nocifensive responses, from 15.1 to $\sim 4 \mathrm{gm}$ (Fig. 2B) (Sham surgery, involving only incision and no retraction, caused only a brief (2-3d) and small $(\sim 2-3 \mathrm{~g})$ drop in threshold, as previously reported by Buvanendram et al. [49], data not shown.). The lowest threshold was reached by POD5-7 and, despite the small decrease in allodynic area that occurred by POD36 (Fig. 1), there was no significant change in the threshold after POD10 (Fig. 2B). In subsequent experiments, male rats have been monitored for 9 weeks after thoracotomy and have shown no signs of recovery of threshold (data not shown).
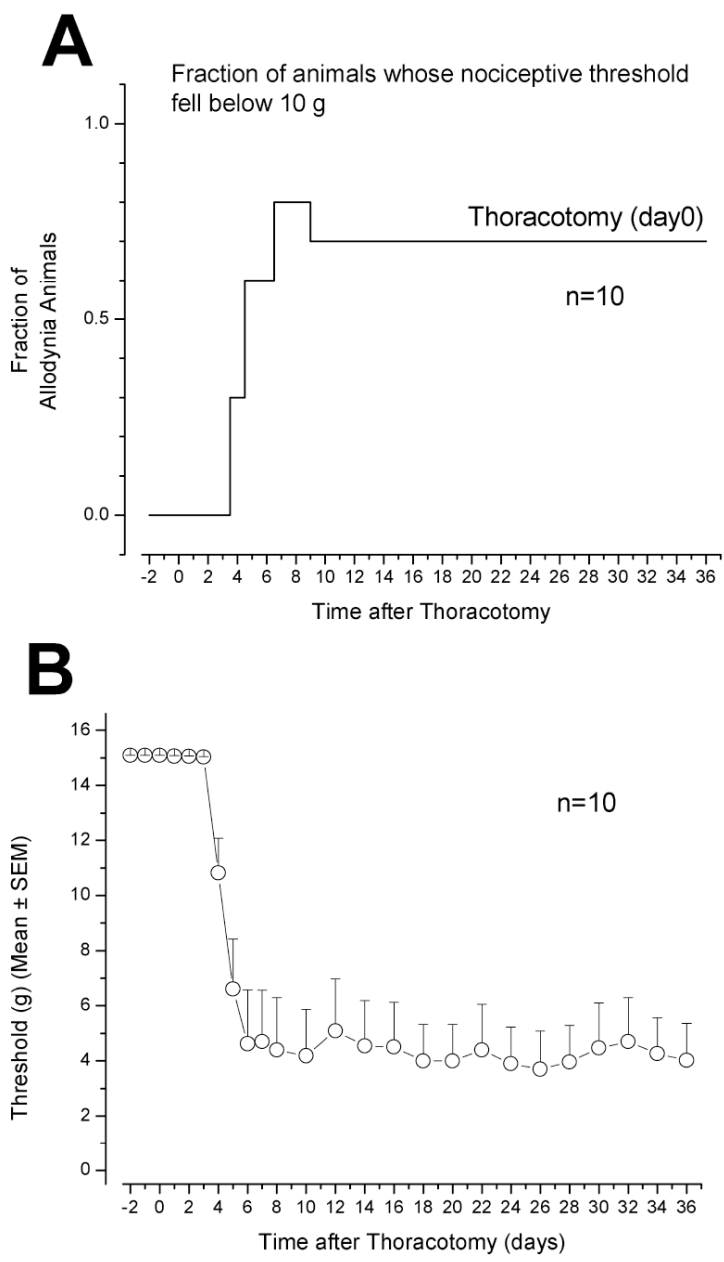

Fig. (2). A). The fraction of rats in a group $(n=10)$ that received thoracotomy and retraction, that showed any form of nocifensive response to probing with a $10 \mathrm{~g}$ VFH. Note the delay in the onset of increase in this "allodynic fraction". B). The average threshold of force to cause a nocifensive response falls from $15 \mathrm{gm}$ (assigned to animals that did not respond to this force, the maximum used, or did respond only to this force and none lower) to approximately 4 gms, with 2 days delay before any detectable drop in threshold. 
Spatial variation in threshold after thoracotomy. The drop in average threshold after thoracotomy and retraction, measured over the entire dorso- thoracolumbar region, can be further resolved by mapping the spatial distribution of thresholds. In intact rats during the preoperative test period those rats that do respond (with only local cutaneous muscle contraction, Grade I, see Methods and below) have the lowest thresholds at the most caudal locations, and nearer the midline (Fig. 3A). For this cohort of rats (which were a different population than those used for experiments of Figs. 1 and 2), the lowest thresholds in intact skin are 7-8 g. The threshold distribution pattern changes after surgery, with the lowest thresholds, now less than $2 \mathrm{~g}$, located in the rostral region around the incision, and on both ipsi- and contralateral sides (Fig. 3B). There was a consistent and significant difference between thresholds here, with the contralateral side's threshold (0.9-1 g) lower than the ipsilateral side's (1.3-1.9 g). Perhaps this difference is due to local, unilateral denervation and subsequent sensory loss around the incision on the ipsilateral side.

Intensity grading of responses is altered by thoracotomy. In preoperative testing, behavior was characterized as Grade 0 in about one third of the rats, and Grade I in the other two thirds (Fig. 4). In none of the three groups of rats ( $\mathrm{n}=8$ in each) that have been characterized this way has there been pre-operative behavior greater than Grade I when the VFH force was limited to $15 \mathrm{gm}$. Thoracotomy and rib retraction led to the appearance of the more intense behavior of Grades II and III, with ca. $80 \%$ of the rats showing Grade III responses for the cohort used for Fig. 4's experiments, the remainder with the milder behavior of Grade I (14\%) or with no response (Grade 0, 7\%). This shift to the higher grades of response was constant when tested at PODs 5, 14 and 36 (data not shown).

Thoracotomy and rib retraction induces ATF-3 expression in DRG neurons. To examine the extent of nerve damage in the thoracotomy model, we investigated the expression of ATF-3, a transcription factor that is only expressed in DRG neurons with axonal injury [56]. As expected, ATF-3 is not expressed in DRG neurons under normal conditions (Fig. 5a). However, thoracotomy and rib retraction induced ATF-3 expression in many neurons in the T4 DRG at POD10 (Fig. 5b), indicating extensive axonal injury. Both small and large diameter cells in these ganglia express ATF-3. Double staining with NF-200 shows that ATF-3 occurs in both myelinated (NF-200-positive) and unmyelinated (NF-200-negative) neurons (Fig. 5c). Taken together, these results show that incision and rib-retraction produces marked nerve injury in both unmyelinated and myelinated fibers, measured at a time when mechanohypersensitivity had reached its maximum value.

\section{SYSTEMIC MORPHINE TEMPORARILY REVERSES THE SIGNS OF ALLODYNIA AFTER THORA- COTOMY}

Intraperitoneal injection of morphine $(2.5 \mathrm{mg} / \mathrm{kg})$, a dose without sedative effects in rats [55], partially reverses tactile hyperalgesia after thoracotomy and retraction (Fig. 6). At 30 minutes - 2 hours after morphine injection the average threshold was above that of post-thoracotomy rats that received i.p. saline, but still below that of the pre-operative threshold of this cohort (Fig. 6A). This partial, transient relief by morphine fell to insignificant levels by $3 \mathrm{hr}$ after injection.
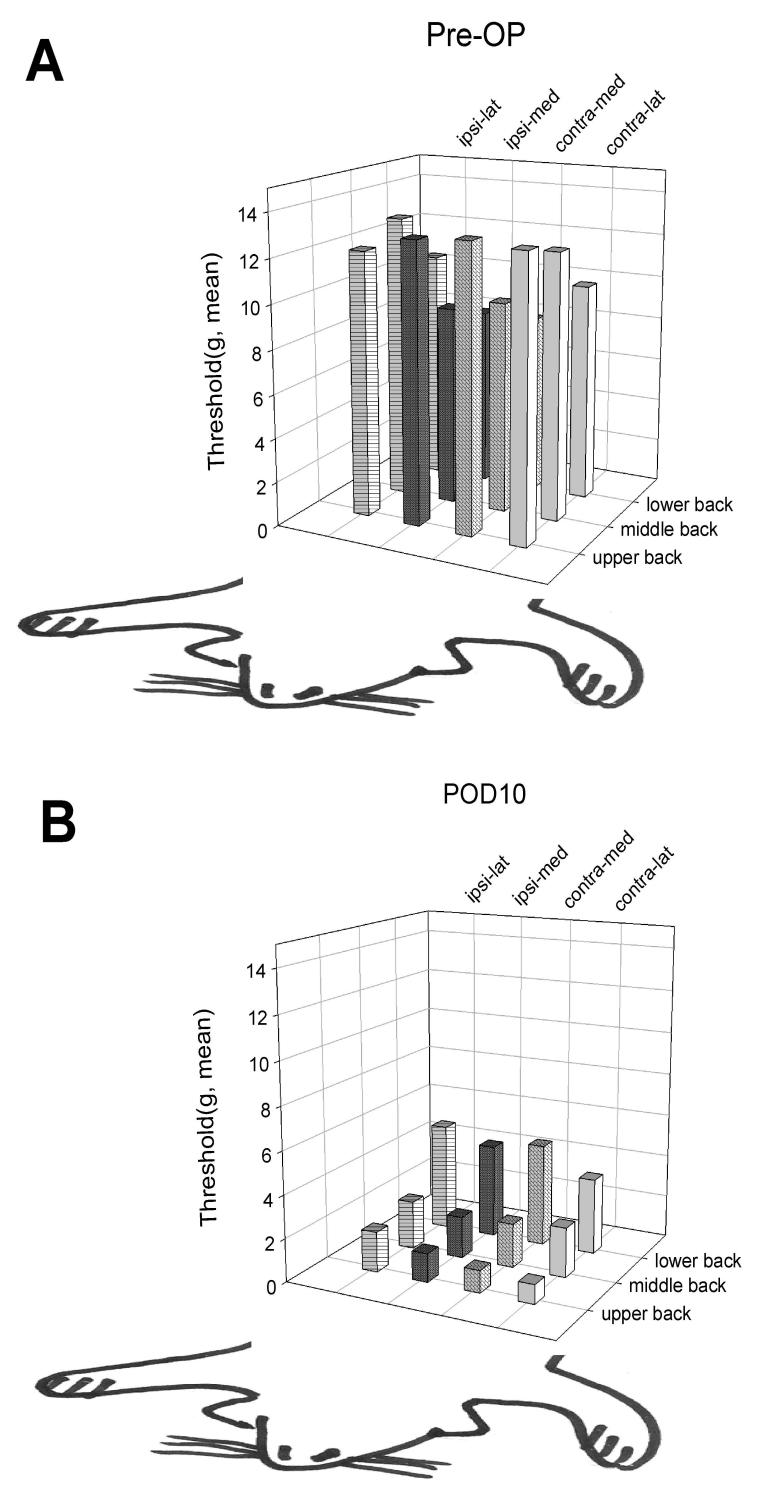

Fig. (3). Anatomical distribution of mechanical thresholds for nocifensive responses on the back (A). before and (B). 10 days after thoracotomy, when maximum hypersensitivity was reached; $n=8$ (see Fig. 2). Note that the lowest thresholds before thoracotomy are on the lower back (A) but after surgery they are on the upper back, near the incision (see Fig. 1). The anatomical directions are opposite in $\mathbf{A}$ and $\mathbf{B}$ so that the bar heights are not obscured (after Nara et al. [50]).

Examination of the responses of individual animals showed the variation in the degree and time-course of threshold change after morphine, with two rats fully recovering to pre-operative levels and two not changing at all (Fig. 6B). Interestingly, those two rats that were morphine insensitive (rats number 3 and 8 ) also had lower preoperative threshold values, determined just before surgery (POD0), than the morphine-responsive rats had at that time. There was no correlation between the post-operative 

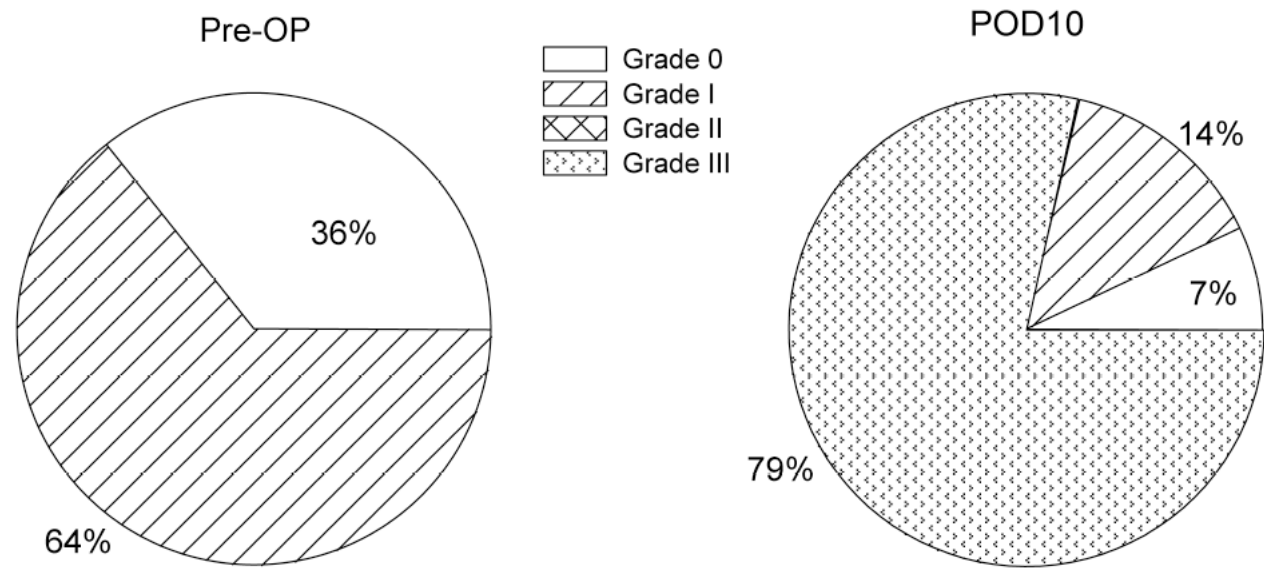

Fig. (4). Distribution of nocifensive responses at threshold among the 4 grades of the Qualitative Hyperalgesic Profile before and after thoracotomy. About one-third of the unoperated rats are unresponsive to any stimulus at or below the $15 \mathrm{~g}$ VFH. All responses of unoperated rats consists of Grade I behavior, which required a $15 \mathrm{~g}$ VFH (see Fig. 2B). When post-thoracotomy hypersensitivity has reached its steady level, 10 days after surgery (see Fig. 2B), almost $80 \%$ of the rats respond to the (now much lower) threshold stimulus with Grade III behavior, and fewer than $10 \%$ of the rats show no response at all, even to a $15 \mathrm{gm} \mathrm{VFH.}(\mathrm{n}=14)$.

ATF-3

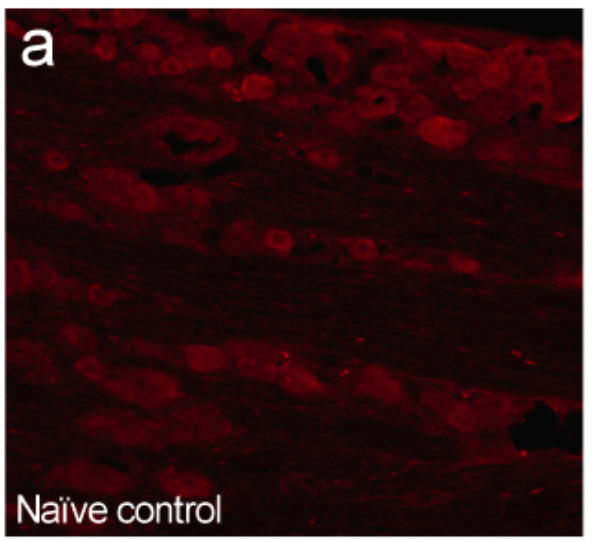

ATF-3

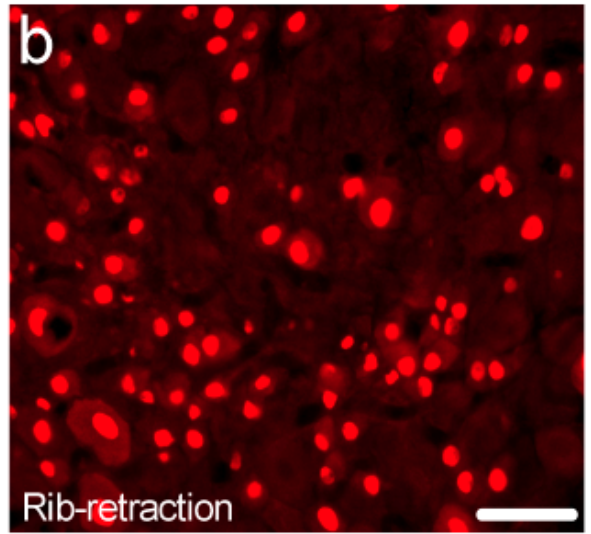

ATF-3 / NF-200

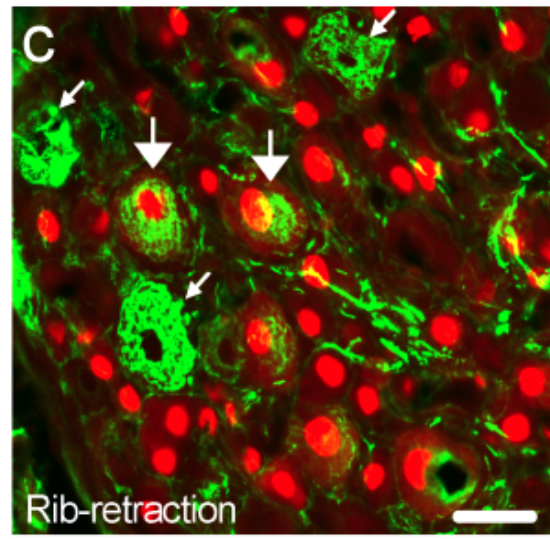

Fig. (5). (a, b) ATF-3 immunofluorescence in the T4 DRG of a naïve control rat (a) and an allodynic rat 10 days after rib-retraction (b). Scale, $50 \mu \mathrm{m}$. (c) Double immunofluorescence of ATF-3 (red) and NF-200 (green) in the T4 DRG of an allodynic rat after rib-retraction. ATF-3 is partially co-localized with NF-200. Large and small allows indicate double-labeled and NF-200 singly-labeled neurons, respectively. Scale, $25 \mu \mathrm{m}$ a,b and $50 \mu \mathrm{m}$ for c.

threshold, measured on POD13 just before morphine administration, and the responsiveness to morphine.

Spatial resolution over the back skin of these threshold changes shows that all areas reached maximum relief at the same time, $30 \mathrm{~min}$, after morphine (Supplemental Fig. 1). The medial ipsilateral region of the upper back, which is the area around the incision retraction, was normalized to about the same threshold as the lateral region of the caudal, contralateral side. Regression of relief occurred in parallel in all 12 test areas over the following 1-4 hrs (Supplemental Fig. 1).

The morphine-induced reversal of tactile hypersensitivity was paralleled by a shift in the Qualitative Hyperalgesia Profile. Eight rats that showed Grade III behavior at POD13 were characterized for their response to i.p. morphine (Supplemental Fig. 2). At 30 min post-morphine, the peak time of threshold reversal, only $25 \%$ of the rats remained at Grade III; about 40\% showed Grade II and the remaining ca. $40 \%$ showed Grades 0 and I, the same distribution as seen in pre-operative rats (see Fig. 4). This distribution in the Qualitative Hyperalgesic Profile gradually shifted back to the post-operative pattern over the next 3-4 hours, although the distribution had not been fully restored to its premorphine pattern at $4 \mathrm{~h}$, despite the complete return of the tactile threshold to pre-morphine level at that time (Supplemental Fig. 2).

\section{DISCUSSION}

Comparison of experimental and clinical postthoracotomy pain. The rat model used here simulates closely the surgical procedures common to many clinical thoracotomies. We made no effort to avoid nerve damage, and this may be why we found that about $70 \%$ of the operated rats developed mechano-hypersensitivity [49, 51, 52], not unlike the incidence reported for humans [20, 21, 27, 28]. Since the intent here was to establish a symptomatology like that in humans, for the testing of potential preventive or treatment therapeutics, this high incidence is a desirably 
efficient feature. Rats showed an un-abating hyperalgesic pattern for the 5 weeks over which we tested them, in agreement with the 40 days of constantly low threshold reported earlier [49]. The model thus also has the features of a chronic pain paradigm, although the behavior has not been followed for longer than 2 months, the duration of pain that generally qualifies it as "chronic."

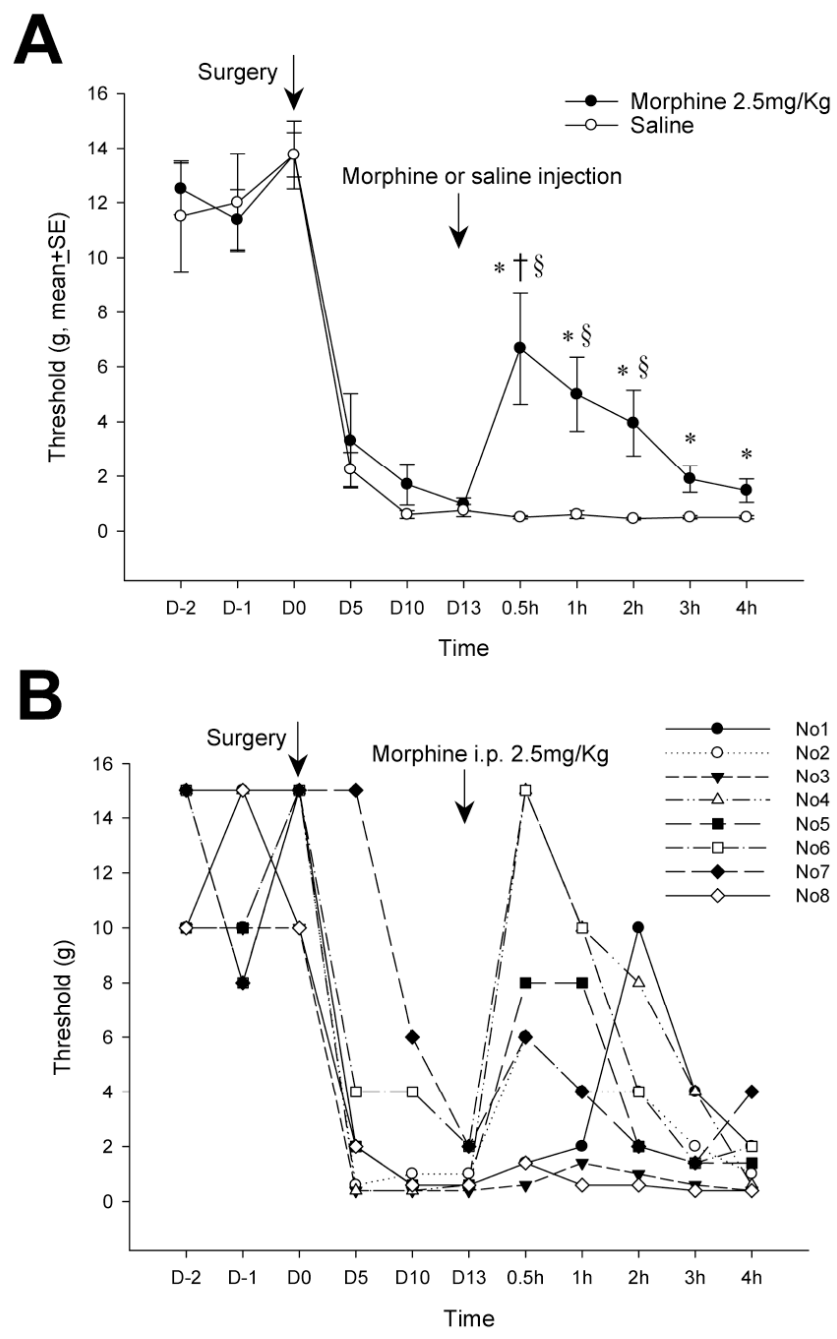

Fig. (6). (A). Systemic morphine injected on post-operative day 13 raises the mechanical threshold for nocifensive response back towards pre-operative levels. $* \mathrm{P}<0.05$ compared to the preoperative level (repeated measures ANOVA) shows that the relief by morphine never reaches the pre-operative, baseline condition. $\S$ $\mathrm{P}<0.05$ for comparison on each respective day, of morphine-treated rats with those treated with saline (One way ANOVA), shows that morphine relieved hypersensitivity at $0.5,1$ and $2 \mathrm{~h}$ after its injection. $\uparrow \mathrm{P}<0.05$ compared to the threshold at POD 13 (repeated measure ANOVA), just before morphine injection, shows that relief is only significant for the $0.5 \mathrm{~h}$ after morphine. (B). Responses to morphine of threshold in individual rats in the group that is averaged in (Fig. 6A). $(n=8$ in both morphine and saline groups).

There are also several salient differences between the model and the clinical findings. In the rat, as reported previously [49], there is a delay of 3-4 days after surgery before hypersensitivity is detected. By comparison, hypersensitivity after skin or skin muscle incision in rats [7, $62,63]$ appears within less than one hour, although that from skin-muscle incision plus retraction takes 1-2 days [19]. Ligation of the intercostal nerves produced hypersensitivity at least as early as post-operative day 1 [50]. The reasons for this delay of several days in the onset of hyperalgesic responsiveness after thoracotomy is not known.

Post-thoracotomy pain and nerve injury. A substantial proportion of clinical post-thoracotomy pain has been characterized as "neuropathic," with both incident and resting pain present [22, 30, 64-66]. Neurophysiological investigations of these patients show conduction deficits [67, 68], often including total conduction block [69], in the nerves at either margin of the incision/retraction zone [40, $41,68,39]$. These findings imply partial or total transection of the nerve, with the subsequent hyperalgesia due to either denervation or to the formation of mechano-sensitive neuromas in the mass of re-growing proximal axon endings [70]. Neuro-histological studies in experimental models of thoracotomy reveal extensive myelinated nerve damage around the wound area in allodynic rats, but absent in rats that did not develop allodynia [49], although the clinical literature has contradictory correlations reported between nerve conduction changes and post-thoracotomy pain [68, $69,41]$.

The immunocytochemical results in the current paper show that both myelinated and non-myelinated nerve fibers are injured in rats suffering hypersensitivity after thoracotomy. The contribution of these different fiber types to the induced mechanosensitivity is not known, but it is noteworthy that pre-operative treatment of intercostal nerves with resiniferatoxin, which is known to selectivity inactivate the TRPV-1 expressing non-myelinated fibers, did not prevent post-thoracotomy pain [52].

Intentional and accidental nerve injury, usually with accompanying inflammation, is known to result in a high incidence of chronic pain [70-72]. In support of this, surgical procedures adopted to minimize nerve injury during thoracotomy, for example by changing the way that the ribs were sutured during closing, has markedly reduced the occurrence of chronic pain [32-37]. Epidural nerve blocks have also been effective in preventing development of this pain $[24,42]$, supporting the concept that an initial large afferent nociceptor discharge is critical in sensitizing the central nervous system to a state that will maintain chronic pain, with some tonic input from the periphery required. In animal models, use of systemic local anesthetic, present at the low concentrations that are known to blunt the ectopic discharges characteristic of injured nerves [73, 74], reduces chronic post-thoracotomy pain [51]. However, it may be wrong to conclude that prolonged post-surgical pain is a consequence of nerve injury per se [50]. Retraction of soft tissue, skin and muscle, that traps and stretches the saphenous nerve leads to the development of tactile and cold allodynia in the ipsilateral distal limb that persists for $\sim 4$ weeks, with evidence of no or very little neural damage at the retraction site, or of activation of injury signaling factors, i.e., ATF-3, in the related sensory ganglion [19]. So prolonged pain may occur without nerve injury, but nerve injury almost certainly causes prolonged pain. 
The Qualitative Hyperalgesic Profile. One goal of this work was to define behaviors in the rat that were direct indicators of perceived pain. The withdrawal of distal extremities or the twitching of subcutaneous muscles can be attributed to purely spinal processes and the afferent signals that produce them may not reach the brain in a form that is perceived and has an affective component that corresponds to human pain. We found a number of novel responses to the thoracotomy procedure that were not present when intact, pre-operative rats were stimulated by these same or much stronger tactile stimuli, yet did appear when the skin on that area of the back was pinched forcefully with forceps. These behaviors were categorized into grades that formed a population distribution, called the Qualitative Hyperalgesia Profile (QHP), in order to characterize "pain-indicating" behaviors. That these signs were actually indicative of "pain" was verified by treatment with systemic morphine, which transiently removed most of the higher grades in the QHP in parallel with a transient reversal of mechanohypersensitivity. Therefore, we propose that this behavioral test is a valid indicator of perceived pain, with both objective and subjective, affective components, after thoracotomy and that it can be modified to provide a similar index for pain after other surgical procedures.

\section{CONFLICT OF INTEREST}

The author(s) confirm that this article content has no conflicts of interest.

\section{ACKNOWLEDGEMENT}

The authors thank Mr. James Bell, Brigham and Women's Hospital Anesthesiology Department, for substantial support and advice with figures, and Dr. Xiaoxia Liu for statistical help.

\section{SUPPLEMENTARY MATERIALS}

Supplementary material is available on the publishers web site along with the published article.

\section{REFERENCES}

[1] Dirks J, Moiniche S, HIlsted KL, Dahl JB. Mechanisms of postoperative pain: Clinical indications for a contribution of central neuronal sensitization. Anesthesiology 2002; 97: 1591-6.

[2] Dworkin RH, McDermott MP, Raja SN. Preventing chronic postsurgical pain: how much of a difference makes a difference? Anesthesiology 2010; 112: 516-8.

[3] Kehlet H, Jensen TS, Woolf CJ. Persistent postsurgical pain: risk factors and prevention. Lancet 2006; 367: 1618-25.

[4] Landau R, Kraft JC, Flint LY, et al. An experimental paradigm for the prediction of Post-Operative Pain (PPOP). J Vis Exp 2010; (35): 1671.

[5] Macrae WA. Chronic post-surgical pain: 10 years on. Br J Anaesth 2008; 101: 77-86.

[6] Kissin I, Gelman S. Chronic postsurgical pain: still a neglected topic? J Pain Res 2012; 5: 473-89.

[7] Banik RK, Subieta AR, Wu C, Brennan TJ. Increased nerve growth factor after rat plantar incision contributes to guarding behavior and heat hyperalgesia. Pain 2005; 117: 68-76.

[8] Clark JD, Qiao Y, Li X, Shi X, Angst MS, Yeomans DC. Blockade of the complement C5a receptor reduces incisional allodynia, edema, and cytokine expression. Anesthesiology 2006; 104: 127482.

[9] Mujenda FH, Duarte AM, Reilly EK, Strichartz GR. Cutaneous endothelin-A receptors elevate post-incisional pain. Pain 2007; 133: 161-73.
[10] Sahbaie P, Shi X, Guo TZ, et al. Role of substance P signaling in enhanced nociceptive sensitization and local cytokine production after incision. Pain 2009; 145: 341-9.

[11] Zahn PK, Pogatzki-Zahn EM, Brennan TJ. Spinal administration of MK-801 and NBQX demonstrates NMDA-independent dorsal horn sensitization in incisional pain. Pain 2005; 114: 499-510.

[12] Zahn PK, Sluka KA, Brennan TJ. Excitatory amino acid release in the spinal cord caused by plantar incision in the rat. Pain 2002; 100: 65-76.

[13] Banik RK, Brennan TJ. Trpv1 mediates spontaneous firing and heat sensitization of cutaneous primary afferents after plantar incision. Pain 2009; 141: 41-51.

[14] Wen Y-R, Suter MR, Ji R-R, et al. Activation of p38 mitogenactivated protein kinase in spinal microglia contributes to incisioninduced mechanical allodynia. Anesthesiology 2009; 110: 155-65.

[15] Gao YJ, Ji RR. Activation of JNK pathway in persistent pain. Neurosci Lett 2008; 437: 180-3.

[16] Huang L, Gao Y-J, Wang J, Strichartz G. Shifts in cell-type expression accompany a diminishing role of spinal p38MAPKinase activation over time during prolonged postoperative pain. Anesthesiology 2011; 115: 1281-90.

[17] Ito N, Obata H, Saito S. Spinal microglial expression and mechanical hypersensitivity in a postoperative pain model: comparison with a neuropathic pain model. Anesthesiology 2009; 111: $640-8$.

[18] Ji RR, Gereau RWT, Malcangio M, Strichartz GR. MAP kinase and pain. Brain Res Rev 2009; 60: 135-48.

[19] Flatters SJL. Characterization of a model of persistent postoperative pain evoked by skin/muscle incision and retraction (SMIR). Pain 2008; 135: 119-30.

[20] Dajczman E, Gordon A, Dreisman H, Wolkove N. Long-term postthoracotomy pain. Chest 1991; 99: 270.

[21] Gottschalk A, Ochroch EA. Clinical and demographic characteristics of patients with chronic pain after major thoracotomy. Clin J Pain 2008; 24: 708-16.

[22] Guastella V, Mick G, Soriano C, et al. A prospective study of neuropathic pain induced by thoracotomy: incidence, clinical description, and diagnosis. Pain 2011; 152: 74-81.

[23] Karmakar MK, Ho AM. Postthoracotomy pain syndrome. Thorac Surg Clin 2004; 14: 345-52.

[24] Obata H, Saito S, Fujita N, Fuse Y, Ishizaki K, Goto F. Epidural block with mepivacaine before surgery reduces long-term postthoracotomy pain. Can J Anaesth 1999; 46: 1127-32.

[25] Ochroch EA, Gottschalk A, Troxel AB, Farrar JT. Women suffer more short and long-term pain than men after major thoracotomy. Clin J Pain 2006; 22: 491-8.

[26] Wang HT, Liu W, Luo AL, Ma C, Huang YG. Prevalence and risk factors of chronic post-thoracotomy pain in Chinese patients from Peking Union Medical College Hospital. Chin Med J (Engl) 2012; 125: 3033-8.

[27] Katz J, Jackson M, Kavanagh BP, Sandler AN. Acute pain after thoracic surgery predicts long-term post-thoracotomy pain. Clin J Pain 1996; 12: 50-5.

[28] Khelemsky Y, Noto CJ. Preventing post-thoracotomy pain syndrome. Mt Sin J Med 2012; 79: 133-9.

[29] Maguire MF, Ravenscroft A, Beggs D, Duffy JP. A questionnaire study investigating the prevalence of the neuropathic component of chronic pain after thoracic surgery. Eur J Cardiothorac Surg 2006; 29: 800-5.

[30] Steegers MA, Snik DM, Verhagen AF, van der Drift MA, WilderSmith $\mathrm{OH}$. Only half of the chronic pain after thoracic surgery shows a neuropathic component. J Pain 2008; 9: 955-61.

[31] Wildgaard K, Ravn J, Kehlet H. Chronic post-thoracotomy pain: a critical review of pathogenic mechanisms and strategies for prevention. Eur J Cardiothorac Surg 2009; 36: 170-80.

[32] Bolotin G, Buckner GD, Jardine NJ, et al. A novel instrumented retractor to monitor tissue-disruptive forces during lateral thoracotomy. J Thorac Cardiovasc Surg 2007; 133: 949-54.

[33] Cerfolio RJ, Bryant AS, Patel B, Bartolucci AA. Intercostal muscle flap reduces the pain of thoracotomy: a prospective randomized trial. J Thorac Cardiovasc Surg 2005; 130(4): 987-93.

[34] Cerfolio RJ, Price TN, Bryant AS, Sale Bass C, Bartolucci AA. Intracostal sutures decrease the pain of thoracotomy. Ann Thorac Surg 2003; 76(2): 407-11; discussion 411-12.

[35] Garcia-Tirado J, Rieger-Reyes C. Suture techniques of the intercostal space in thoracotomy and their relationship with post- 
thoracotomy pain: a systematic review. Arch Bronconeumol 2011; 48: $22-8$.

[36] Landreneau RJ, Pigula F, Luketich JD, et al. Acute and chronic morbidity differences between muscle-sparing and standard lateral thoracotomies. J Thorac Cardiovasc Surg 1996; 112: 1346-50.

[37] Koop O, Gries A, Eckert S, et al. The role of intercostal nerve preservation in pain control after thoracotomy. Eur J Cardiothorac Surg 2013; 43(4): 808-12.

[38] Lee JI, Kim GW, Park KY. Intercostal bundle-splitting thoracotomy reduces chronic post-thoracotomy pain. Thorac Cardiovasc Surg 2007; 55: 401-2.

[39] Maguire MF, Latter JA, Mahajan R, Beggs FD, Duffy JP. A study exploring the role of intercostal nerve damage in chronic pain after thoracic surgery. Eur J Cardiothorac Surg 2006; 29: 873-9.

[40] Miyazaki T, Sakai T, Tsuchiya T, et al. Assessment and follow-up of intercostal nerve damage after video-assisted thoracic surgery. Eur J Cardiothorac Surg 2011; 39: 1033-9.

[41] Rogers ML, Henderson L, Mahajan RP, Duffy JP. Preliminary findings in the neurophysiological assessment of intercostal nerve injury during thoracotomy. Eur J Cardiothorac Surg 2002; 21: 298301.

[42] Bong CL, Samuel M, Ng JM, Ip-Yam C. Effects of preemptive epidural analgesia on post-thoracotomy pain. J Cardiothorac Vasc Anesth 2005; 19: 786-93.

[43] Chen Y, Liao Z, Li H. Reducing the prevalence of chronic postthoracotomy pain syndrome: is total intravenous anaesthesia superior to inhalation anaesthesia? Eur J Cardiothorac Surg 2013; 43(3): 659 .

[44] Senturk M, Ozcan PE, Talu GK, et al. The effects of three different analgesia techniques on long-term postthoracotomy pain. Anesth Analg 2002; 94: 11-5.

[45] Song JG, Shin JW, Lee EH, et al. Incidence of post-thoracotomy pain: a comparison between total intravenous anaesthesia and inhalation anaesthesia. Eur J Cardiothorac Surg 2012; 41: 1078-82.

[46] Wheatley GH, 3rd, Rosenbaum DH, Paul MC, et al. Improved pain management outcomes with continuous infusion of a local anesthetic after thoracotomy. J Thorac Cardiovasc Surg 2005; 130: 464-8.

[47] Strichartz G, Pastijn E, Sugimoto K. Neural physiology and local anesthetic action, In: Cousins MJ, Carr DB, Horlocker TT, Bridenbaugh, PO. Eds. Neural Blockade in Clinical Anesthesia and Pain Medicine. USA, PA: Walters Kluwer-Lippincott Williams and Wilkins 2009; pp. 26-47.

[48] Kristensen AD, Pedersen TA, Hjortdal VE, Jensen TS, Nikolajsen L. Chronic pain in adults after thoracotomy in childhood or youth. Br J Anaesth 2010; 104: 75-9.

[49] Buvanendran A, Kroin JS, Kerns JM, Nagalla SN, Tuman KJ. Characterization of a new animal model for evaluation of persistent postthoracotomy pain. Anesth Analg 2004; 99: 1453-60.

[50] Nara T, Saito S, Obata H, Goto F. A rat model of postthoracotomy pain: behavioural and spinal cord NK-1 receptor assessment. Can J Anaesth 2001; 48: 665-76.

[51] Shin JW, Pancaro C, Wang CF, Gerner P. Low-dose systemic bupivacaine prevents the development of allodynia after thoracotomy in rats. Anesth Analg 2008; 107: 1587-91.

[52] Shin JW, Pancaro C, Wang CF, Gerner P. The effects of resiniferatoxin in an experimental rat thoracotomy model. Anesth Analg 2010; 110: 228-32.

[53] Mogil JS, Davis KD, Derbyshire SW. The necessity of animal models in pain research. Pain 2010; 151: 12-7.

[54] Rice AS, Cimino-Brown D, Eisenach JC, et al. Animal models and the prediction of efficacy in clinical trials of analgesic drugs: a critical appraisal and call for uniform reporting standards. Pain 2008; 139: 243-7.
[55] Granmo M, Jensen T, Schouenborg J. Nociceptive Transmission to Rat Primary Somatosensory Cortex - Comparison of Sedative and Analgesic Effects. PLoS ONE 2013; 8(1): e53966.

[56] Tsujino H, Kondo E, Fukuoka T, et al. Activating transcription factor 3 (ATF3) induction by axotomy in sensory and motoneurons: A novel neuronal marker of nerve injury. Mol Cell Neurosci 2000; 15(2): 170-82.

[57] National Research Council of the National Academies. Guide for the Care and Use of Laboratory Animals. $8^{\text {th }}$ ed. US: Academic Press 2011

[58] Weksler B, Ng B, Lenert J, Burt M. A simplified method for endotracheal intubation in the rat. J Appl Physiol 1994; 76: 1823-5.

[59] Theriault E, Diamond J. Nociceptive cutaneous stimuli evoke localized contractions in a skeletal muscle. J Neurophysiol 1988; 60: 446-62.

[60] Zhuang ZY, Gerner P, Woolf CJ, Ji RR. ERK is sequentially activated in neurons, microglia, and astrocytes by spinal nerve ligation and contributes to mechanical allodynia in this neuropathic pain model. Pain 2005; 114: 149-59.

[61] Zhuang ZY, Wen YR, Zhang DR, et al. A peptide c-Jun N-terminal kinase (JNK) inhibitor blocks mechanical allodynia after spinal nerve ligation: respective roles of JNK activation in primary sensory neurons and spinal astrocytes for neuropathic pain development and maintenance. J Neurosci 2006; 26: 3551-60.

[62] Xu J, Brennan TJ. Comparison of skin incision vs. skin plus deep tissue incision on ongoing pain and spontaneous activity in dorsal horn neurons. Pain 2009; 144: 329-39.

[63] Duarte AM, Pospisilova E, Reilly E, Mujenda F, Hamaya Y, Strichartz, GR. Reduction of post-incisional allodynia by subcutaneous bupivacaine: Findings with a new model in the hairy skin of the rat. Anesthesiology 2005; 103: 113-25.

[64] Katz J, Asmundson GJ, McRae K, Halket E. Emotional numbing and pain intensity predict the development of pain disability up to one year after lateral thoracotomy. Eur J Pain 2009; 13: 870-8.

[65] Hopkins KG, Rosenzweig M. Post-thoracotomy pain syndrome: assessment and intervention. Clin J Oncol Nurs 2012; 16: 365-70.

[66] Wildgaard K, Ringsted TK, Hansen HJ, Peterson RH, Werner MU, Kehlet $\mathrm{H}$. Quantitative sensory testing of persistent pain after video-assisted thoracic surgery lobectomy. Br J Anaesth 2012; 108(1): 126-33.

[67] Wildgaard K, Ringsted TK, Aasvang EK, Ravn J, Werner MU, Kehlet H. Neurophysiological Characterization of Persistent Postthoracotomy Pain. Clin J Pain 2012; 28(2): 136-42.

[68] Benedetti F, Vighetti S, Ricco C, et al. Neurophysiologic assessment of nerve impairment in posterolateral and musclesparing thoracotomy. J Thorac Cardiovasc Surg 1998; 115: 841-7.

[69] Michaelis M. Electrophysiological Characteristics of Injured Peripheral Nerves. Eds. In: Malmberg AB, Chaplan SR, Mechanisms and Mediators of Neuropathic Pain. Basel: Birkhäuser Verlag 2002; pp. 3-16.

[70] Decosterd I, Woolf CJ. Spared nerve injury: an animal model of persistent peripheral neuropathic pain. Pain 2000; 87: 149-58.

[71] DeLeo JA, Yezierski RP. The role of neuroinflammation and neuroimmune activation in persistent pain. Pain 2001; 90: 1-6.

[72] Wall PD, Devor M, Inbal R, et al. Autotomy following peripheral nerve lesions: experimental anaesthesia dolorosa. Pain 1979; 7 : 103-11.

[73] Khodorova A, Meissner K, Leeson S, Strichartz GR. Lidocaine selectively blocks abnormal impulses arising from non-inactivating Na channels. Muscle Nerve 2001; 24: 634-48.

[74] Persaud N, Strichartz GR. Micromolar lidocaine selectively blocks propagating ectopic impulses at a distance from their site of origin. Pain 2002; 99: 333-40.

Received: July 23, 2013

Revised: September 19, 2013

Accepted: September 20,2013

(c) Wang et al.; Licensee Bentham Open.

This is an open access article licensed under the terms of the Creative Commons Attribution Non-Commercial License (http://creativecommons.org/licenses/by-nc/3.0/) which permits unrestricted, non-commercial use, distribution and reproduction in any medium, provided the work is properly cited. 